\title{
Content analysis of library websites of central universities of North East India
}

\author{
Gyanajeet Yumnam $^{1 *}$, Ch. Ibohal Singh ${ }^{2}$ \\ ${ }^{1}$ Research Scholar (UGC-NET), ${ }^{2}$ Associate Professor and Head, Dept. of Library and Information Science, Manipur University (Central \\ University), Canchipur, Imphal, Manipur, India \\ *Corresponding Author: Gyanajeet Yumnam \\ Email: ygyanajeet@gmail.com
}

\begin{abstract}
Library websites play an essential role in collecting and sharing information or sources for users. Today it is a common platform to interact with users without their behavior. A website survey certainly helps us to identify and analyse available content to obtain rich and suitable information by evaluating the current website's improvement. The present study evaluates the contents of the nine central university library websites of the North East India. A checklist has been developed to collect library facilities, library collections, university webpage accessibility, link searches, and the retrieval interface. The purpose of this article is to help website developers, and administrators better understand the current state of these university websites so that they may provide more informative, up-to-date information, authentic information, user-friendly, and dynamic methods to users based on their needs.
\end{abstract}

Keywords: Content analysis, Central University, Library websites, North East India.

\section{Introduction}

With the advent of the World Wide Web (WWW) and its application, the world has become a much smaller place, in fact, a global village. There has been a significant change among the people of different sections in accessing information and communicating with each other. The new generation lives in a digital world, using smartphones, computers, and electronic devices linked through social media like Facebook, YouTube, Instagram, Twitter, etc. These devices and applications provide an irreplaceable source of education and self-education in the form of new literacy. There are different social networking sites, including blogging, image and video sharing, and mobile connectivity. In addition, LIS services may help promote Library and Information Centers services. The library website serves as a vital gateway to library services. Therefore, usability analysis and assessment of library websites are of paramount importance. "The designing of an educational website needs to be more focused on usability and shows that different perspectives derived from the area of specialization, age and gender, and category give a different evaluation" (Shukla, 2017).

\section{Brief details about Central Universities in India}

An act of Parliament establishes central Universities in India. They are under the Department of Higher Education's purview in the Ministry of Education (MoE). These universities have been categorized under Central Government, given that the MoE allocates the funds through UGC. There are 54 Central Universities established in India as of $31^{\text {st }}$ March 2021 (UGC, 2021). Out of these 54 Universities, 9 Universities are selected from the Northeastern region of India for the present study.

\section{Review of Literature}

There is no dearth of literature on the study of content analysis of library websites. Kumar and Mir (2017), in their Content Analysis of Central Universities Library Websites of
Central Region of India, found that Central Universities have a rich site of collections of E-books, E-journals, list of EJournal, E- database in all four central Universities and every central university library websites provides the facility of daily news and having contacts on their library websites. Shukla (2017), in his content analysis, found that most of the selected central universities libraries websites of the Northeastern region of India were ignoring their usability features. There was numerous scope of further improvement in Web 2.0 tools, web searching features, library committee, website update, website map, multilingual website option, etc. Shukla and Tripathi (2010) made a comparative study of 20 central universities and 19 institutes of national importance, including Indian Institutes of Technology (IITs) and Indian Institutes of Management (IIMs) academic libraries from India using a method to measure Overall Website Performance Calculation (OWPC) and Criteria-wise Website Performance. Haridasan and Uwesh (2014) revealed that most university library websites in India provide an informative link to contacts, news, and events.

\section{Significance of the study}

The study investigates the contents of the nine library websites of central universities of the region. The library websites of these central universities' help their users provide relevant literature/material/services and serve as a vital platform for marketing the information products and services of the university libraries.

\section{Objectives}

The present study has been taken up with the following objectives:

1. To know the location and establishment of central universities in the Northeastern region of India.

2. To know the general information provided by their library websites.

3. To know the non-book material and e-resources provided by them. 
4. To identify social networking sites implemented in the websites.

\section{Methodology and Scope}

The data have been collected through survey and observation from the nine websites of the libraries of the nine central universities of India, namely: (a) Tezpur University (Assam), (b) North-Eastern Hill University (Meghalaya), (c) Mizoram University (Mizoram), (d) Assam University (Assam), (e) Sikkim University (Sikkim), (f) Manipur University (Manipur), (g) Rajiv Gandhi University (Arunachal Pradesh), (h) Tripura University (Tripura), and (i) Nagaland University (Nagaland). A checklist has been developed and used as a tool for collecting data from the websites. The data has been analysed about the websites' content with a specific response to general information, selection, facilities, services and best practices, and web 2.0 software. The data for the study has been collected from 5 June to 10 October 2021.

\section{Data Analysis}

Universities under study

The following Table 1 shows the name and year of establishment of the Central Universities covered in the present study. It also depicts the different URLs of selected websites of institutions and their libraries. It is also seen that Manipur University Library has no separate and particular library websites but, the other libraries maintained their library websites.

Table 1: Central Universities under study

\begin{tabular}{|c|c|c|c|c|}
\hline $\begin{array}{l}\text { University } \\
\text { Name }\end{array}$ & State & $\begin{array}{l}\text { Estd. } \\
\text { Year }\end{array}$ & URL & Library URL \\
\hline Tezpur University (TZU) & Assam & 1994 & www.tezu.ernet.in & http://www.tezu.ernet.in/Library/ \\
\hline $\begin{array}{l}\text { North-Eastern Hill University } \\
\text { (NEHU) }\end{array}$ & Meghalaya & 1973 & $\begin{array}{l}\text { https://www.nehu.a } \\
\text { c.in }\end{array}$ & https://www.nehu.ac.in/library/ \\
\hline Mizoram University(MZU) & Mizoram & 2001 & https://mzu.edu.in & https://lib.mzu.edu.in/ \\
\hline Assam University (AU) & Assam & 1989 & www.aus.ac.in & http://www.aus.ac.in/library/ \\
\hline Sikkim University (SKU) & Sikkim & 2006 & $\begin{array}{l}\text { https://cus.ac.in > } \\
\text { index.php }\end{array}$ & https://library.cus.ac.in \\
\hline Manipur University (MU) & Manipur & 1980 & $\begin{array}{l}\text { www.manipuruniv. } \\
\text { ac.in }\end{array}$ & www.manipuruniv.ac.in > library \\
\hline Rajiv Gandhi University (RGU) & $\begin{array}{l}\text { Arunachal } \\
\text { Pradesh }\end{array}$ & 1984 & https://rgu.ac.in/ & https://rgu.ac.in/library-home/ \\
\hline Tripura University (TU) & Tripura & 1987 & $\begin{array}{l}\text { https://www.tripura } \\
\text { univ.ac.in/ }\end{array}$ & $\begin{array}{l}\text { https://www.tripurauniv.ac.in/Home } \\
\text { /CentralLibraryIndex }\end{array}$ \\
\hline Nagaland University (NU) & Nagaland & 1989 & $\begin{array}{l}\text { https://nagalanduni } \\
\text { versity.ac.in/ }\end{array}$ & $\begin{array}{l}\text { https://library.nagalanduniversity.ac } \\
\text {.in/ }\end{array}$ \\
\hline
\end{tabular}

$* U R L=$ Uniform Resource Locator

\section{General Information of the Universities}

As Table 2 shows, the general information available in library websites include information about the library, library, copyright, working hour, membership, library rule, staff section, and library section. In addition, most libraries websites are found to be given information on 'About library'.

Table 2: General Information available on the websites

\begin{tabular}{|r|l|c|c|c|c|c|c|c|c|c|}
\hline S. No. & General Information & AU & MU & MZU & NEHU & TU & NU & RGU & SKU & TZU \\
\hline 1. & About Library & $\checkmark$ & $\checkmark$ & $\checkmark$ & $\checkmark$ & $\checkmark$ & $\checkmark$ & $\checkmark$ & $\checkmark$ & $\checkmark$ \\
\hline 2. & Vision \&Mission & $\mathbf{x}$ & $\mathbf{x}$ & $\mathbf{x}$ & $\mathbf{x}$ & $\checkmark$ & $\checkmark$ & $\mathbf{x}$ & $\mathbf{x}$ & $\checkmark$ \\
\hline 3. & Objectives & $\mathbf{x}$ & $\mathbf{x}$ & $\mathbf{x}$ & $\mathbf{x}$ & $\mathbf{x}$ & $\mathbf{x}$ & $\checkmark$ & $\mathbf{x}$ & $\mathbf{x}$ \\
\hline 4. & Site Map/Location & $\checkmark$ & $\checkmark$ & $\checkmark$ & $\mathbf{x}$ & $\mathbf{x}$ & $\checkmark$ & $\checkmark$ & $\checkmark$ & $\checkmark$ \\
\hline 5. & Library Hour & $\checkmark$ & $\checkmark$ & $\checkmark$ & $\checkmark$ & $\checkmark$ & $\checkmark$ & $\checkmark$ & $\checkmark$ & $\checkmark$ \\
\hline 6. & Membership & $\checkmark$ & $\checkmark$ & $\checkmark$ & $\checkmark$ & $\checkmark$ & $\checkmark$ & $\checkmark$ & $\checkmark$ & $\checkmark$ \\
\hline 7. & Library Rules & $\checkmark$ & $\mathbf{x}$ & $\checkmark$ & $\mathbf{x}$ & $\checkmark$ & $\mathbf{x}$ & $\mathbf{x}$ & $\mathbf{x}$ & $\checkmark$ \\
\hline 8. & Library Committee & $\checkmark$ & $\mathbf{x}$ & $\checkmark$ & $\mathbf{x}$ & $\checkmark$ & $\checkmark$ & $\checkmark$ & $\checkmark$ & $\checkmark$ \\
\hline 9. & News and Events & $\checkmark$ & $\mathbf{x}$ & $\checkmark$ & $\mathbf{x}$ & $\mathbf{x}$ & $\mathbf{x}$ & $\checkmark$ & $\checkmark$ & $\checkmark$ \\
\hline 10. & Photo Gallery & $\checkmark$ & $\mathbf{x}$ & $\checkmark$ & $\checkmark$ & $\checkmark$ & $\checkmark$ & $\checkmark$ & $\checkmark$ & $\checkmark$ \\
\hline 11. & Website Copyright & $\checkmark$ & $\mathbf{x}$ & $\checkmark$ & $\checkmark$ & $\checkmark$ & $\checkmark$ & $\checkmark$ & $\checkmark$ & $\checkmark$ \\
\hline 12. & Library Staff & $\checkmark$ & $\mathbf{x}$ & $\checkmark$ & $\checkmark$ & $\checkmark$ & $\mathbf{x}$ & $\checkmark$ & $\checkmark$ & $\checkmark$ \\
\hline 13. & Library Sections & $\checkmark$ & $\checkmark$ & $\checkmark$ & $\checkmark$ & $\checkmark$ & $\checkmark$ & $\checkmark$ & $\checkmark$ & $\checkmark$ \\
\hline 14. & Services & & & $\checkmark$ & & \\
\hline
\end{tabular}




\begin{tabular}{|c|l|c|c|c|c|c|c|c|c|c|}
\hline 15. & FAQ & $\checkmark$ & $\mathbf{x}$ & $\checkmark$ & $\checkmark$ & $\checkmark$ & $\mathbf{x}$ & $\mathbf{x}$ & $\checkmark$ & $\checkmark$ \\
\hline 16. & Visitors Count & $\checkmark$ & $\mathbf{x}$ & $\mathbf{x}$ & $\mathbf{x}$ & $\checkmark$ & $\checkmark$ & $\checkmark$ & $\mathbf{x}$ & $\checkmark$ \\
\hline 17. & Institutional/Digital Repository & $\checkmark$ & $\mathbf{x}$ & $\checkmark$ & $\mathbf{x}$ & $\checkmark$ & $\checkmark$ & $\mathbf{x}$ & $\checkmark$ & $\checkmark$ \\
\hline 18. & eResources & $\checkmark$ & $\checkmark$ & $\checkmark$ & $\checkmark$ & $\checkmark$ & $\checkmark$ & $\checkmark$ & $\checkmark$ & $\checkmark$ \\
\hline 19. & WebOPAC & $\checkmark$ & $\mathbf{x}$ & $\checkmark$ & $\checkmark$ & $\checkmark$ & $\checkmark$ & $\checkmark$ & $\checkmark$ & $\checkmark$ \\
\hline 20. & e-Library/Remote Access & $\checkmark$ & $\checkmark$ & $\checkmark$ & $\checkmark$ & $\checkmark$ & $\checkmark$ & $\mathbf{x}$ & $\mathbf{x}$ & $\checkmark$ \\
\hline 21. & Useful Links & $\checkmark$ & $\checkmark$ & $\checkmark$ & $\checkmark$ & $\checkmark$ & $\checkmark$ & $\mathbf{x}$ & $\checkmark$ & $\checkmark$ \\
\hline
\end{tabular}

Information about the Library

The data presented in Table 3 and figure 1 reveals that the nine libraries websites have given information on about library, library hour, membership, services and e-resources with (100\%) and information about library rules, website copyright, library staff, web OPAC and useful links were provided in the eight libraries (88.89\%). Site Map/Location, News and Events, Library Sections and e-Library/Remote Access were found in seven library websites with $77.78 \%$. Only one library website, i.e. RGU, gives information about the library's objectives on their websites.

Table 3: General information about the library

\begin{tabular}{|l|c|c|}
\hline General Information & No. of Library & \% \\
\hline About Library & 9 & 100.00 \\
\hline Vision \& Mission & 3 & 33.33 \\
\hline Objectives & 1 & 11.11 \\
\hline Site Map/Location & 7 & 77.78 \\
\hline Library Hour & 9 & 100.00 \\
\hline Membership & 9 & 100.00 \\
\hline Library Rules & 8 & 88.89 \\
\hline Library Committee & 4 & 44.44 \\
\hline News and Events & 7 & 77.78 \\
\hline Photo Gallery & 5 & 55.56 \\
\hline Website Copyright & 8 & 88.89 \\
\hline Library Staff & 8 & 88.89 \\
\hline Library Sections & 7 & 77.78 \\
\hline Services & 9 & 100.00 \\
\hline FAQ & 6 & 66.67 \\
\hline Visitors Count & 5 & 55.56 \\
\hline Institutional/Digital Repository & 6 & 66.67 \\
\hline eResources & 9 & 100.00 \\
\hline Web OPAC & 8 & 88.89 \\
\hline e-Library/Remote Access & 7 & 77.78 \\
\hline Useful Links & 8 & 88.89 \\
\hline
\end{tabular}

*Tick sign ' $\checkmark$ ' (Available) and cross sign ' $\boldsymbol{x}$ ' (Not Available)

\section{On Collection Development}

Table 4 shows the library collections of the universities on their websites. It is observed that all the university libraries have books, periodicals, E-Journals, reference sources, thesis, dissertation, and newspapers. In contrast, all libraries have not a collection of Photographs. Tezpur University is the only libraries having a collection of manuscripts and e-patents. In addition, MZU has a collection of Projects.

Table 4: Information about library collection

\begin{tabular}{|c|l|c|c|c|c|c|c|c|c|c|}
\hline S. No. & Library Collection & AU & MU & MZU & NEHU & TU & NU & RGU & SKU & TZU \\
\hline 1. & Books & $\checkmark$ & $\checkmark$ & $\checkmark$ & $\checkmark$ & $\checkmark$ & $\checkmark$ & $\checkmark$ & $\checkmark$ & $\checkmark$ \\
\hline 2. & Periodicals & $\checkmark$ & $\checkmark$ & $\checkmark$ & $\checkmark$ & $\checkmark$ & $\checkmark$ & $\checkmark$ & $\checkmark$ & $\checkmark$ \\
\hline 3. & eBooks & $\checkmark$ & $\checkmark$ & $\checkmark$ & $\checkmark$ & $\checkmark$ & $\checkmark$ & $\checkmark$ & $\checkmark$ & $\checkmark$ \\
\hline 4. & EJournals & $\checkmark$ & $\checkmark$ & $\checkmark$ & $\checkmark$ & $\checkmark$ & $\checkmark$ & $\checkmark$ & $\checkmark$ & $\checkmark$ \\
\hline 5. & E-Courses & $\mathbf{x}$ & $\mathbf{x}$ & $\mathbf{x}$ & $\mathbf{x}$ & $\mathbf{x}$ & $\mathbf{x}$ & $\mathbf{x}$ & $\mathbf{x}$ & $\checkmark$ \\
\hline 6. & E-Database & $\checkmark$ & $\checkmark$ & $\checkmark$ & $\checkmark$ & $\checkmark$ & $\mathbf{x}$ & $\mathbf{x}$ & $\mathbf{x}$ & $\checkmark$ \\
\hline 7. & E-Patents & $\mathbf{x}$ & $\mathbf{x}$ & $\mathbf{x}$ & $\mathbf{x}$ & $\mathbf{x}$ & $\mathbf{x}$ & $\mathbf{x}$ & $\mathbf{x}$ & $\checkmark$ \\
\hline 8. & Audio/Video Collection & $\mathbf{x}$ & $\mathbf{x}$ & $\checkmark$ & $\checkmark$ & $\checkmark$ & $\checkmark$ & $\checkmark$ & $\checkmark$ & $\checkmark$ \\
\hline
\end{tabular}




\begin{tabular}{|c|l|c|c|c|c|c|c|c|c|c|}
\hline 9. & Reference sources & $\checkmark$ & $\checkmark$ & $\checkmark$ & $\checkmark$ & $\checkmark$ & $\checkmark$ & $\checkmark$ & $\checkmark$ & $\checkmark$ \\
\hline 10. & Back volumes of journals & $\mathbf{x}$ & $\checkmark$ & $\checkmark$ & $\mathbf{x}$ & $\checkmark$ & $\checkmark$ & $\checkmark$ & $\checkmark$ & $\checkmark$ \\
\hline 11. & Photographs & $\mathbf{x}$ & $\mathbf{x}$ & $\mathbf{x}$ & $\mathbf{x}$ & $\mathbf{x}$ & $\mathbf{x}$ & $\mathbf{x}$ & $\mathbf{x}$ & $\mathbf{x}$ \\
\hline 12. & Newspapers & $\checkmark$ & $\checkmark$ & $\checkmark$ & $\checkmark$ & $\checkmark$ & $\checkmark$ & $\checkmark$ & $\checkmark$ & $\checkmark$ \\
\hline 13. & Thesis & $\checkmark$ & $\checkmark$ & $\checkmark$ & $\checkmark$ & $\checkmark$ & $\checkmark$ & $\checkmark$ & $\checkmark$ & $\checkmark$ \\
\hline 14. & Dissertations & $\checkmark$ & $\checkmark$ & $\checkmark$ & $\checkmark$ & $\checkmark$ & $\checkmark$ & $\checkmark$ & $\checkmark$ & $\checkmark$ \\
\hline 15. & Reports & $\mathbf{x}$ & $\checkmark$ & $\mathbf{x}$ & $\mathbf{x}$ & $\mathbf{x}$ & $\mathbf{x}$ & $\mathbf{x}$ & $\mathbf{x}$ & $\checkmark$ \\
\hline 16. & Manuscripts & $\mathbf{x}$ & $\mathbf{x}$ & $\mathbf{x}$ & $\mathbf{x}$ & $\mathbf{x}$ & $\mathbf{x}$ & $\mathbf{x}$ & $\mathbf{x}$ & $\checkmark$ \\
\hline 17. & Projects & $\mathbf{x}$ & $\mathbf{x}$ & $\checkmark$ & $\mathbf{x}$ & $\mathbf{x}$ & $\mathbf{x}$ & $\mathbf{x}$ & $\mathbf{x}$ & $\mathbf{x}$ \\
\hline 18. & Administrative Documents & $\mathbf{x}$ & $\checkmark$ & $\mathbf{x}$ & $\mathbf{x}$ & $\mathbf{x}$ & $\mathbf{x}$ & $\mathbf{x}$ & $\checkmark$ & $\checkmark$ \\
\hline
\end{tabular}

*Tick sign ' $\checkmark$ ' (Available) and cross sign ' $\boldsymbol{x}$ ' (Not Available)

\section{Use of Social Networking Sites}

Here, Social Networking tools implemented and adapted by the websites of the libraries under study are being analysed. As seen in Table 5, among the nine library websites, AU and TZU are implemented most social networking tools to interact with their users and provide opportunities to participate in libraries' activities. However, being not having library websites, MU is far less than other universities in implementing these social media tools.

Table 5: Social Networking Tools Implemented University Libraries

\begin{tabular}{|c|c|c|c|c|c|c|c|c|c|c|c|c|c|c|c|}
\hline \multirow[t]{2}{*}{ S. No. } & \multirow[t]{2}{*}{ University } & \multicolumn{14}{|c|}{ Social Media Interfaces } \\
\hline & & 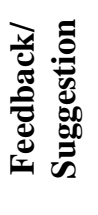 & 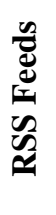 & 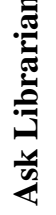 & $\begin{array}{l}\underbrace{n}_{\tilde{E}} \\
\stackrel{\tilde{E}}{\tilde{E}}\end{array}$ & $\overrightarrow{\tilde{\Xi}}$ & 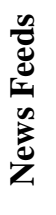 & $\stackrel{\mathscr{6}}{00}$ & 刍 & 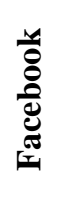 & 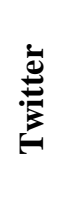 & 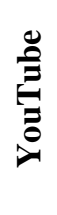 & 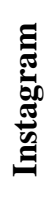 & $\begin{array}{l}+ \\
\frac{0}{00} \\
0 \\
0 \\
0\end{array}$ & 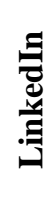 \\
\hline 1. & $\mathrm{AU}$ & $\checkmark$ & $\checkmark$ & $\checkmark$ & $\checkmark$ & $x$ & $\checkmark$ & $x$ & $\checkmark$ & $\checkmark$ & $\checkmark$ & $\checkmark$ & $x$ & $\checkmark$ & $\checkmark$ \\
\hline 2. & MU & $x$ & $x$ & $x$ & $x$ & $x$ & $x$ & $x$ & $\checkmark$ & $x$ & $x$ & $x$ & $x$ & $x$ & $x$ \\
\hline 3. & MZU & $\checkmark$ & $x$ & $\checkmark$ & $\checkmark$ & $x$ & $\checkmark$ & $x$ & $\checkmark$ & $\checkmark$ & $\checkmark$ & $\checkmark$ & $\checkmark$ & $x$ & $x$ \\
\hline 4. & NU & $x$ & $x$ & $\checkmark$ & $\checkmark$ & $x$ & $x$ & $x$ & $\checkmark$ & $\checkmark$ & $\checkmark$ & $\checkmark$ & $x$ & $x$ & $x$ \\
\hline 5. & NEHU & $\checkmark$ & $x$ & $x$ & $\checkmark$ & $x$ & $x$ & $x$ & $\checkmark$ & $x$ & $x$ & $x$ & $x$ & $x$ & $x$ \\
\hline 6. & RGU & $x$ & $x$ & $x$ & $\checkmark$ & $x$ & $\checkmark$ & $x$ & $\checkmark$ & $\checkmark$ & $\checkmark$ & $\checkmark$ & $x$ & $x$ & $\checkmark$ \\
\hline 7. & SKU & $x$ & $x$ & $x$ & $\checkmark$ & $\checkmark$ & $\checkmark$ & $x$ & $\checkmark$ & $\checkmark$ & $x$ & $x$ & $x$ & $x$ & $x$ \\
\hline 8. & TZU & $\checkmark$ & $x$ & $\checkmark$ & $\checkmark$ & $\checkmark$ & $\checkmark$ & $\checkmark$ & $\checkmark$ & $\checkmark$ & $\checkmark$ & $\checkmark$ & $x$ & $\checkmark$ & $x$ \\
\hline 9. & $\mathrm{TU}$ & $\checkmark$ & $x$ & $x$ & $\checkmark$ & $x$ & $\checkmark$ & $x$ & $\checkmark$ & $\checkmark$ & $\checkmark$ & $x$ & $x$ & $x$ & $x$ \\
\hline
\end{tabular}

*Tick sign ' $\checkmark$ ' (Available) and cross sign ' $\boldsymbol{x}$ ' (Not Available)

Table 6: Social Networking Tools adapted by the Universities

\begin{tabular}{|l|c|c|}
\hline Social Networking Tools & No. of University & \% \\
\hline Feedback/Suggestion & 5 & 55.56 \\
\hline RSS Feeds & 1 & 11.11 \\
\hline Ask Librarian & 4 & 44.44 \\
\hline Contact Us & 8 & 88.89 \\
\hline Chat & 2 & 22.22 \\
\hline News Feed & 6 & 66.67 \\
\hline Blogs & 1 & 11.11 \\
\hline Email & 9 & 100.00 \\
\hline Facebook & 7 & 77.78 \\
\hline Twitter & 6 & 66.67 \\
\hline YouTube & 5 & 55.56 \\
\hline Instagram & 1 & 11.11 \\
\hline Google+ & 2 & 22.22 \\
\hline LinkedIn & 2 & 22.22 \\
\hline
\end{tabular}


As shown in table 6 among the social networking tools 'Email' is the highest adopted tool in all the nine university library websites with $100 \%$, followed by 'Contact Us' with $88.89 \%$. Social Media tools 'Facebook' is adopted in 7 universities with $77.78 \%$, 'News Feed' and 'Twitter' with $66.67 \%$ each, and so on.

\section{Results and Conclusion}

The study has revealed us many clues on the contents of the websites of these libraries.

1. All the nine Central Universities of Northeastern India have a rich collection of books, periodicals, e-books, ejournals, reference sources, newspapers, these, and dissertations.

2. Almost all the library websites have a social media tool, i.e. Email and Contact Us.

3. It is also found that $100 \%$ of the central library websites and URLs have information about the library, library hour, membership, services and e-resources.

Lastly, the study shows an overview of the contents available on all websites of central universities of the region. It is also observed that the university library websites are an excellent medium to know what is available in the library or not without going in the library; if the material does not exist in any form, how can the university take it as a loan from other universities. It is in this context that the present study has shown many aspects of the issue. In order to reflect changes in user behavior, technology, and IT resources, the information on the university library website needs to be periodically evaluated and analysed, using the contents, structure and design of the website, as needed by the user in this digital era.

\section{Conflict of Interest}

None

\section{Source of Funding \\ None}

\section{References}

1. https://www.ugc.ac.in/centraluniversity.aspx.

2. Shukla A. Usability Analysis of Central Universities Libraries Websites of North-East India: An Evaluative Study. $J$ Adv Res Libr Inf Sci 2017;4(4):37-41.

3. Kumar S., Mir M. S. Content Analysis of Central Universities Library Websites of Central Region of India: A Survey. Int J Sci Technol Res. 2017;6(6):283-6.

4. Shukla A, Tripathi A. Establishing content awareness evaluation criteria for library websites: a case study of Indian academic library websites. Ann Libr Inf Stud 2010; 57:403416.

5. Haridasan S, Uwesh M. Content analysis Central library university library websites in India: A study. J Inf Manag 2014;1(2):59-71.

How to cite: Yumnam G, Singh CI. Content analysis of library websites of central universities of North East India. IP Indian J Libr Sci Inf Technol. 2021;6(1):9-13. 